\title{
First numerical experiences with overlap fermions based on the Brillouin kernel
}

\section{Stephan Dürr*}

University of Wuppertal and IAS/JSC Forschungszentrum Jülich, Germany

E-mail: durr (AT) itp.unibe.ch

\section{Giannis Koutsou}

Cyprus Institute, CaSToRC, Nicosia, Cyprus

E-mail: g.koutsou (AT) cyi.ac.cy

Numerical experiences are reported with overlap fermions which employ the Brillouin action as a kernel. After discussing the dispersion relations of both the kernel and the resulting chiral action, some of the physics features are addressed on quenched backgrounds. We find that the overlap with Brillouin kernel is much better localized than the overlap with Wilson kernel. Also a preliminary account is given of the cost of the formulation, in terms of CPU time and memory.

34th International Symposium on Lattice Field Theory 24-30 July 2016

University of Southampton, UK

\footnotetext{
* Speaker.
} 


\section{Brillouin Fermions}

Designing a lattice fermion action with a continuum-like dispersion relation along with good chiral properties for $a m \ll 1$ and small cut-off effects for $a m=O(1)$ remains a challenge. The "perfect action" approach by Peter Hasenfratz and collaborators aimed at this combination of desirable properties [1], and there have been similar attempts since [2, 3]. Also the Brillouin action [4]

$$
D_{\mathrm{B}}(x, y)=\sum_{\mu} \gamma_{\mu} \nabla_{\mu}^{\mathrm{iso}}(x, y)-\frac{a}{2} \triangle^{\mathrm{bri}}(x, y)+m_{0} \delta_{x, y}-\frac{c_{\mathrm{SW}}}{2} \sum_{\mu<v} \sigma_{\mu v} F_{\mu \nu} \delta_{x, y},
$$

where $\nabla_{\mu}^{\text {iso }}$ is a 54-point discretization of the covariant derivative and $\triangle \triangle^{\text {bri }}$ is a 81-point discretization of the covariant laplacian, belongs to the same category (it differs from previous attempts in all coefficients in the stencils being untuned rational numbers). In order to maintain $\gamma_{5}$-hermiticity an average over all $n$-hop paths $(n=2,3,4)$ in the stencil must be taken (cf. Sec. 3 below).
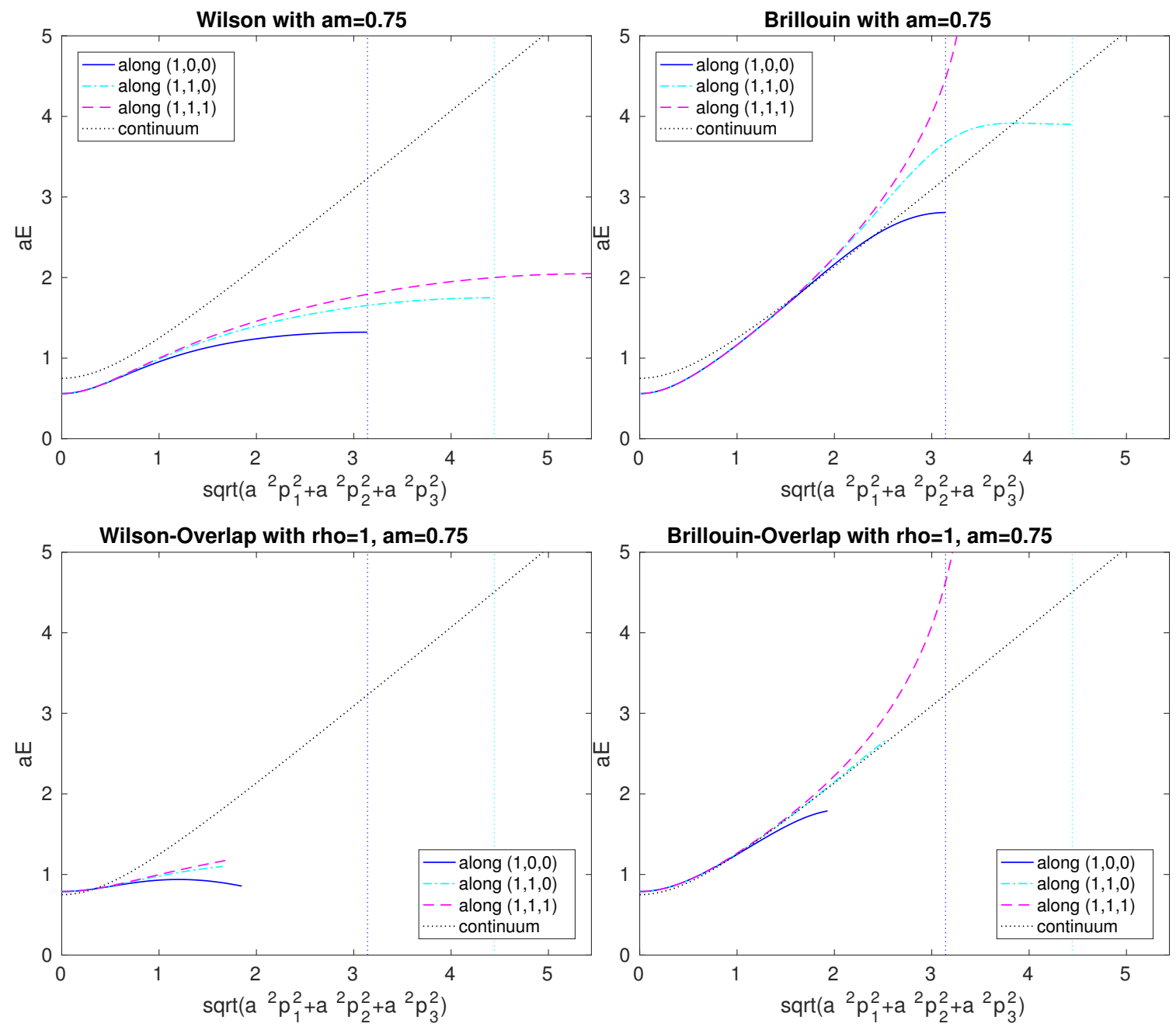

Figure 1: Dispersion relations of the Wilson operator (top left), Brillouin operator (top right), as well as their $\rho=1$ overlap descendents (bottom panels), all at the rather heavy quark mass am $=0.75$. The Brillouin zone ends at $\pi / a$ on axis, and it reaches by a factor $\sqrt{2}$ and $\sqrt{3}$ further out for the other two directions. 


\section{Quark Dispersion Relations}

The Brillouin action at $a m \ll 1$ is known to show a good dispersion relation, both on the quark level (in the weak field limit) [5], and for mesons and baryons (in the interacting theory) [6]. Unfortunately, Fig. 1 shows that this does not carry over to substantial quark masses; the cut-off effect at $\mathbf{p}=0$ is as large as with the Wilson action [5]. Fortunately, applying the overlap procedure is found to (almost) cure this deficiency; the massive dispersion relation stays nearly perfect out to $a\|\mathbf{p}\| \simeq 2$ with the Brillouin kernel, while it breaks down much earlier for the Wilson kernel.

\section{Implementation Details of Kernel Action}

It is important to have an efficient implementation of the Brillouin kernel to start any serious investigation of the Brillouin overlap action. To this end it seems crucial to precompute all offdiagonal links ( 2 contributions to 2-hop, 6 contributions to 3-hop, 24 contributions to 4-hop, always averaged but not necessarily backprojected) and to assemble the relevant 40 links in $W_{v}(x)$ with $v=1 \ldots 40$ to be computed from the smeared gauge field $V_{\mu}(x)$ with $\mu=1 \ldots 4$.

With the object $W_{v}$ in hand the Brillouin flop-count for a matrix-times-vector operation yields 30192 per site, to be compared with the Wilson flop count of 1368 per site (with mass term in both cases but no gauge compression and no e/o-decomposition for Wilson) [7]. Hence, the Brillouinto-Wilson ratio of flops is 22.1, not far from the measured timing ratio 18.5 on a standard 4-core CPU. Similarly, the required memory traffic is 3408 floats for Brillouin and 384 for Wilson, i.e. the Brillouin-to-Wilson ratio of traffic is 8.9 (with several rhs both numbers decrease, but the ratio stays at 8.9) [7]. Overall, the 0.45 bytes/flop sp-ratio of the Brillouin action (as opposed to 1.12 bytes/flop in sp for Wilson) makes it an interesting choice for forthcoming architectures. Further details in F2008 are found in the slides [7], and a complete implementation in C is available at [8].

The measured mat-vec timing ratio $\sim 20$ is mitigated by a factor 4 [9] (due to reduced iteration count and sub-dominance of scalar products) in a solver, so the actual cost increase is a factor $\sim 5$.

\section{Implementation Details of Overlap Action}

Starting from a kernel action at negative mass $-\rho / a$ the massless overlap action is [10]

$$
D_{\mathrm{ovr}, 0}=\frac{\rho}{a}\left\{1+\gamma_{5} \operatorname{sign}\left(\gamma_{5} D_{\mathrm{ker},-\rho / a}\right)\right\}=\frac{\rho}{a}\left\{1+D_{\mathrm{ker},-\rho / a}\left(D_{\mathrm{ker},-\rho / a}^{\dagger} D_{\mathrm{ker},-\rho / a}\right)^{-1 / 2}\right\}
$$

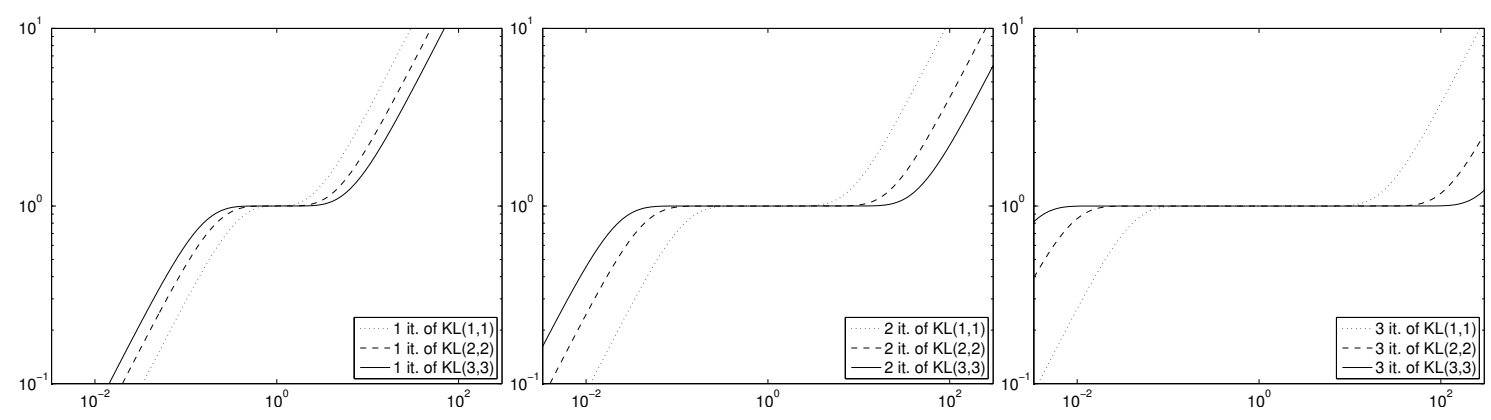

Figure 2: Approximate Kenney-Laub sign functions $f_{11}, f_{22}, f_{33}$ on $] 0, \infty[$ after one (left), two (middle) and three (right) iterations. Note the symmetry about $(1,1)$, and the strictly monotonic behavior. 
and the Kenney-Laub family of approximants to the matrix sign function or inverse square root

$$
f_{11}(x)=x \frac{3+x^{2}}{1+3 x^{2}}, f_{22}(x)=x \frac{5+10 x^{2}+x^{4}}{1+10 x^{2}+5 x^{4}}, f_{33}(x)=x \frac{7+35 x^{2}+21 x^{4}+x^{6}}{1+21 x^{2}+35 x^{4}+7 x^{6}}, f_{44}(x)=f_{11}\left(f_{11}(x)\right)
$$

is a convenient but non-optimal choice with remarkable properties (see Fig. 2 and [9]). This choice does not require any knowledge of the spectral properties of $\gamma_{5} D_{\mathrm{ker},-\rho / a}$ or $D_{\mathrm{ker},-\rho / a}^{\dagger} D_{\mathrm{ker},-\rho / a}$, but if low-lying eigenvalue-eigenvector information is available, it can be used to speed up the CG used in the partial-fraction expansion of an element in eq. 4.2 (see [9] for more details).

\section{Overlap Action Properties}

In Fig. 3 we show the eigenvalue spectra of the Wilson and Brillouin actions, and how they are modified if the fixed-order Kenney-Laub procedure $f_{11}$ from eq. (4.2) is applied on the operator. With the Wilson kernel the physical branch is being pushed towards $\operatorname{Re}(z)=0$ more efficiently than
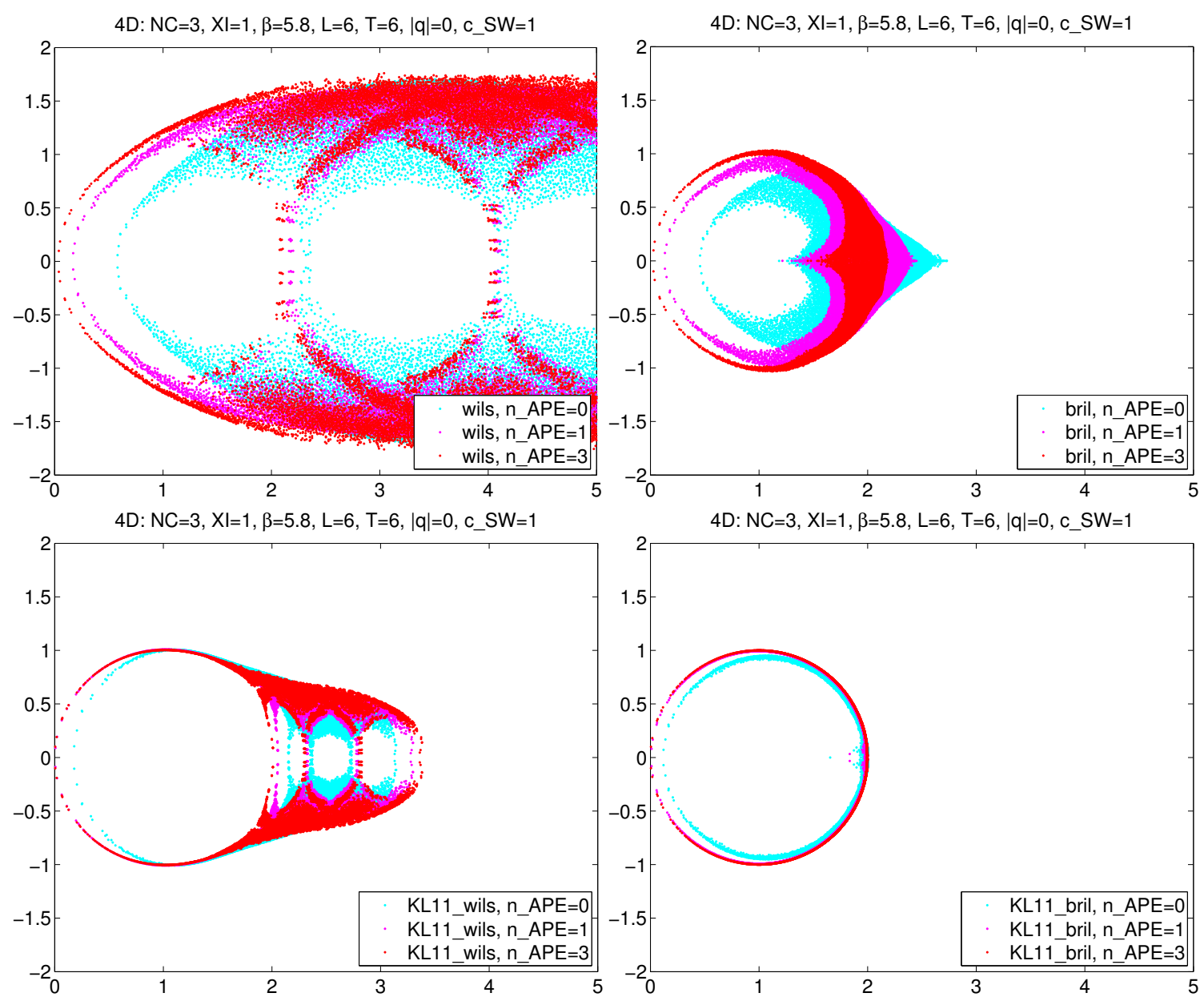

Figure 3: Eigenvalue spectra of the Wilson (top left) and Brillouin (top right) operators on a thermalized quenched $6^{4}$ background, both operators with $c_{\mathrm{SW}}=1$. The three colors refer to three levels of link smearing (cyan for no smearing, magenta for 1 APE step, red for 3 APE steps, in both cases with $\alpha=0.72$ ). The bottom panels display the eigenvalue spectra of the respective $f_{11}$ overlap approximants $(\rho=1)$. 
the remaining 15 branches towards $\operatorname{Re}(z)=2$. With the Brillouin kernel even a single iteration of $f_{11}$ seems to establish an operator with good chiral properties (at least if starting from $D_{\text {ker }}=D_{B}$ with sufficient link smearing). Moreover, since the low-lying physical eigenvalues hardly change, it seems like a self-suggesting idea to use $D_{\mathrm{B}}$ or $D_{\mathrm{W}}$ to precondition the $f_{11}$ approximant [11], and the latter action to precondition a higher-order approximant, e.g. KL44 given by $f_{44}=f_{11}\left(f_{11}\right)$ [9].

\section{First Spectroscopy Results}

An overlap operator evaluated with infinite precision is normal and satisfies the GinspargWilson (GW) relation [12], which was re-discovered by Peter Hasenfratz (2nd work of Ref. [1]).

In Fig. 4 we plot the remnant non-normality against $g_{0}^{2}=6 / \beta$ on volume-matched quenched
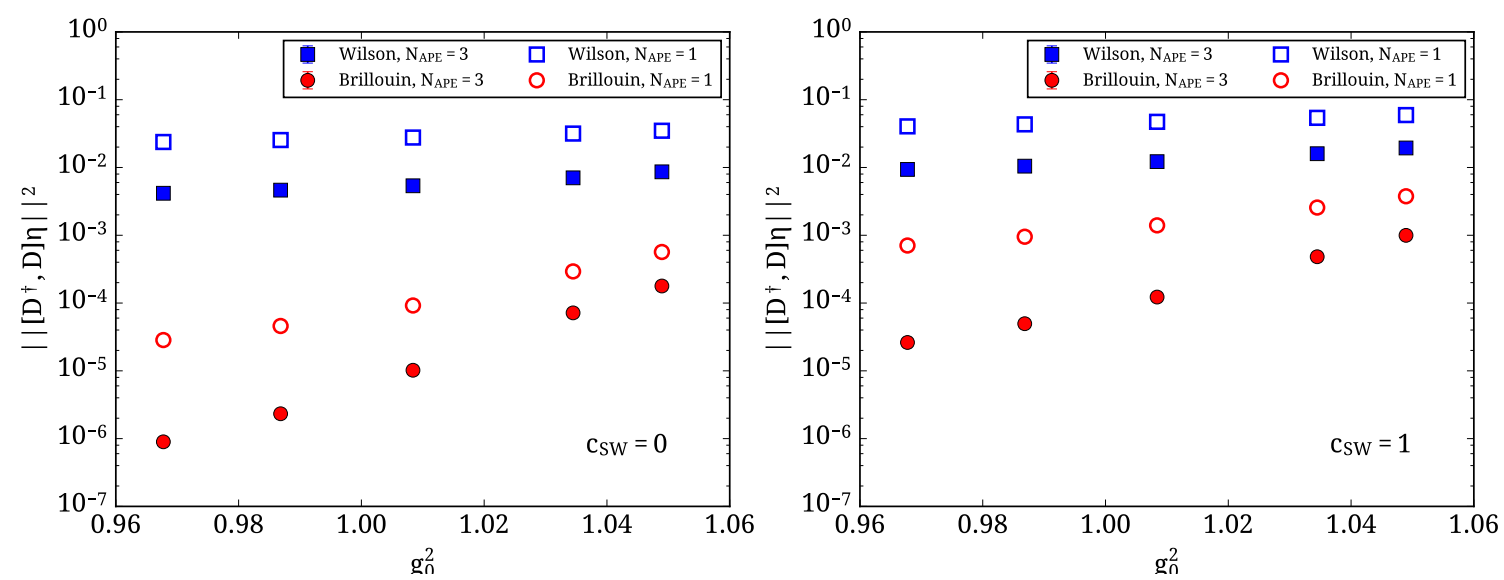

Figure 4: Remnant non-normality of the KL11 overlap approximants based on the Wilson kernel (blue squares) and the Brillouin kernel (red circles) versus $6 / \beta$. Open symbols refer to 1 step of APE smearing, filled symbols to 3 steps. The kernel actions at $\rho=1$ may be unimproved (left) or tree-level improved (right).
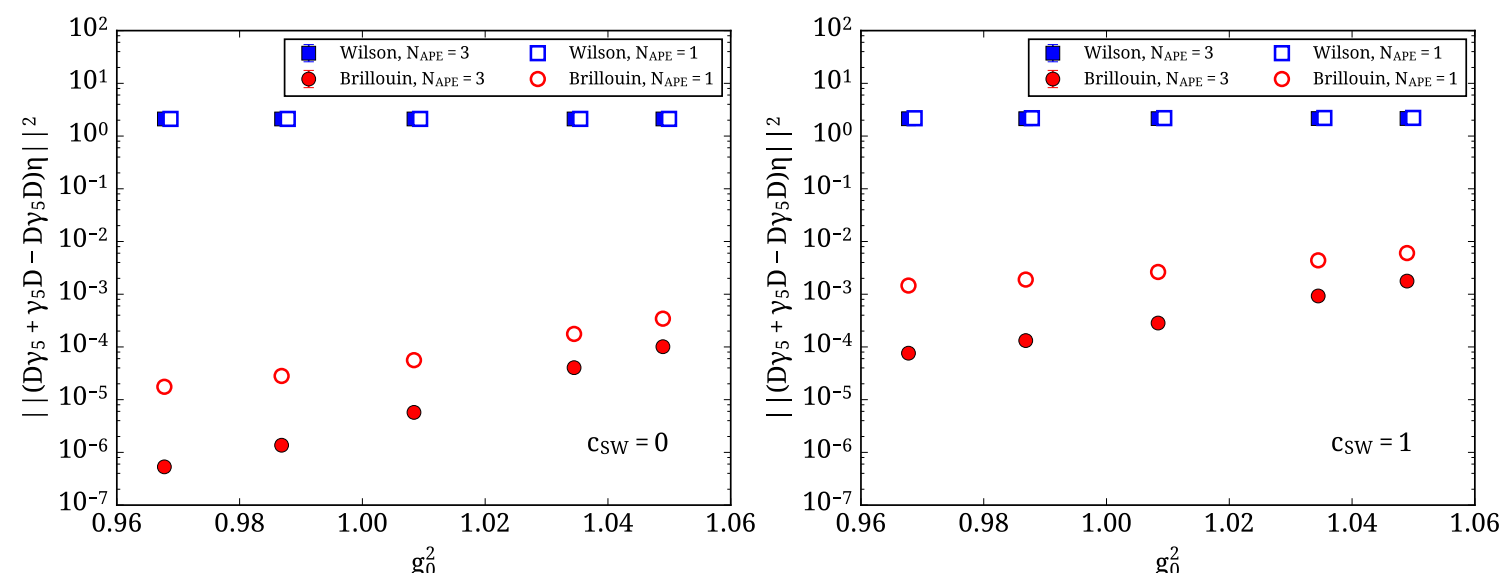

Figure 5: Remnant violation of the GW-relation of the KL11 overlap approximants based on the Wilson kernel (blue squares) and the Brillouin kernel (red circles). Open symbols refer to 1 step of APE smearing, filled symbols to 3 steps. The kernel actions at $\rho=1$ may be unimproved (left) or tree-level improved (right). 
lattices. As the operator norm is not available for such big matrices, we evaluate for Gaussian $\eta$

$$
\left\|\left(D^{\dagger} D-D D^{\dagger}\right) \eta\right\|^{2} /\|\eta\|^{2}
$$

where $D$ denotes the fixed-order KL11 approximant to the massless overlap action based on either the Wilson (blue) or Brillouin (red) kernel, and $\|$.$\| denotes the vector 2-norm. Evidently, the$ version with the Brillouin kernel fares much better; it also benefits more from link smearing.

In Fig. 5 we plot the remnant violation of the GW relation versus $g_{0}^{2}=6 / \beta$ on the same set of lattices. Again, since the operator norm is not available, we evaluate

$$
\left\|\left(D \gamma_{5}+\gamma_{5} D-D \gamma_{5} D\right) \eta\right\|^{2} /\|\eta\|^{2}
$$

where $D$ denotes the fixed-order KL11 approximant to the massless overlap action based on either the Wilson (blue) or Brillouin (red) kernel, and $\|$.$\| denotes the vector 2-norm. Once more, the$ version with the Brillouin kernel performs better and benefits more from link smearing.

From these figures it seems the Wilson kernels with and without improvement yield KL11 overlap actions with comparable properties. On the other hand, among the Brillouin KL11 actions the version with $c_{\mathrm{SW}}=0$ in the kernel seems superior to the version with $c_{\mathrm{SW}}=1$ in the kernel.

The locality properties of the resulting overlap approximants are determined from a vector $\zeta$ which is localized in one grid point, e.g. 0, but Gaussian in color and spinor space. We consider the fall-off in the norm of $\psi=D \zeta$ which roughly follows $\|\psi(x)\| \propto \exp (-\delta|x|)$ where $|x|$ is the Euclidean norm in position space. Fig. 6 shows that the Brillouin overlap falls about twice as steep as the Wilson kernel counterpart. Some meson spectroscopy on $40^{3} \times 64$ lattices is on the slides [7].

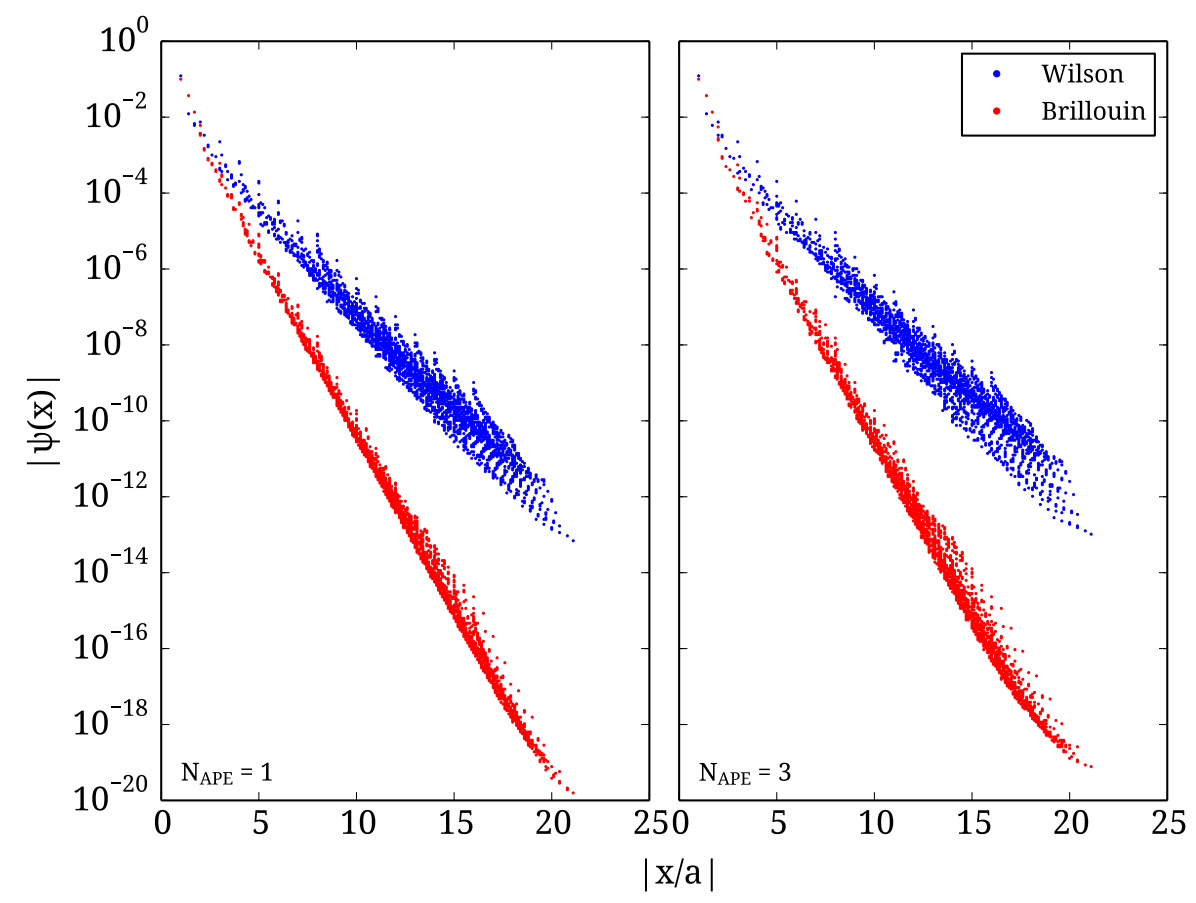

Figure 6: Fall-off (in coordinate space) of the KL11 approximants to the overlap action based on the Wilson (blue) and Brillouin (red) kernels, both at $\rho=1$. Using 1 (left) or 3 (right) steps of APE smearing proves immaterial. Note that no average over different separations $\mathbf{x}$ with joint $\mathbf{x}^{2}$ is taken. 


\section{Summary and Outlook}

The "truly perfect action" (i.e. an action which fulfills the physics requirements of the perfect fermion action as mentioned above and is cheap in terms of CPU requirements) doesn't exist.

The Brillouin kernel improves on the Wilson kernel with respect to the massless free-field dispersion relation [in the sense that $(a E)^{2} \simeq(a \mathbf{p})^{2}$ holds over a larger fraction of the Brillouin zone], but it does not bring any improvement for heavy quark masses [even at $\mathbf{p}=\mathbf{0}$ ]. The overlap procedure (with any kernel) improves both chiral properties at $a m \ll 1$ and heavy-quark properties at $a m=O(1)$. The Brillouin overlap action combines the two ingredients and yields a reasonable approximation to the perfect action as put forth by P. Hasenfratz and other people [1, 2, 3].

Our investigation reveals a better normality and reduced GW-violation of a fixed-order overlap with the Brillouin kernel, in comparison with the Wilson kernel, and the locality in position space is improved. In terms of CPU time, the Brillouin matrix-vector multiplication is roughly a factor 20 more expensive than the Wilson multiplication, but in a solver about a factor 4 comes back from reduced iteration count and related reasons. Using a standard cluster architecture, we have been able to invert Brillouin overlap quarks on lattices of size $40^{3} \times 64$ at reasonable quark masses.

The Brillouin kernel itself shows promising features regarding meson and baryon dispersion relations [6]. We plan to repeat this kind of investigation for the Brillouin-overlap action.

Acknowledgements: Numerical computations were performed on JUROPA at JSC.

\section{References}

[1] T. A. DeGrand, A. Hasenfratz, P. Hasenfratz and F. Niedermayer, Nucl. Phys. B 454, 587 (1995) [hep-lat/9506030]. P. Hasenfratz, Nucl. Phys. Proc. Suppl. 63, 53 (1998) [hep-lat/9709110]. P. Hasenfratz, Nucl. Phys. B 525, 401 (1998) [hep-lat/9802007].

[2] C. Gattringer, Phys. Rev. D 63, 114501 (2001) [hep-lat/0003005]. C. Gattringer, I. Hip and C. B. Lang, Nucl. Phys. B 597, 451 (2001) [hep-lat/0007042].

[3] W. Bietenholz and U. J. Wiese, Nucl. Phys. B 464, 319 (1996) [hep-lat/9510026]. W. Bietenholz and I. Hip, Nucl. Phys. B 570, 423 (2000) [hep-lat/9902019]. W. Bietenholz, Eur. Phys. J. C 6, 537 (1999) [hep-lat/9803023].

[4] S. Durr and G. Koutsou, Phys. Rev. D 83, 114512 (2011) [arXiv:1012.3615 [hep-lat]].

[5] Y. G. Cho, S. Hashimoto, A. Juttner, T. Kaneko, M. Marinkovic, J. I. Noaki and J. T. Tsang, JHEP 1505, 072 (2015) [arXiv:1504.01630 [hep-lat]].

[6] S. Durr, G. Koutsou and T. Lippert, Phys. Rev. D 86, 114514 (2012) [arXiv:1208.6270 [hep-lat]].

[7] See slides at https://conference.ippp.dur.ac.uk/event/470/session/13/contribution/64

[8] See details at https://github.com/g-koutsou/qpb

[9] S. Durr and G. Koutsou, forthcoming.

[10] H. Neuberger, Phys. Lett. B 417, 141 (1998) [hep-lat/9707022]. H. Neuberger, Phys. Lett. B 427, 353 (1998) [hep-lat/9801031].

[11] J. Brannick, A. Frommer, K. Kahl, B. Leder, M. Rottmann and A. Strebel, Numer. Math. (2015) [arXiv:1410.7170 [hep-lat]].

[12] P. H. Ginsparg and K. G. Wilson, Phys. Rev. D 25, 2649 (1982). 